\title{
Characterization of B-cell Receptor Heavy Chain Complementarity- determining Region 3 Repertoire Based on the Differences of Symbiotic Microorganisms in the Gut of Mice
}

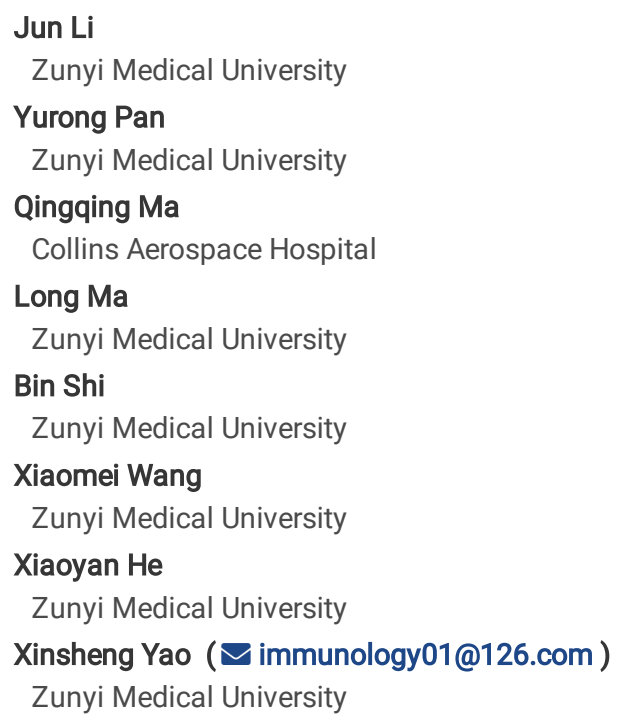

Research article

Keywords: Gut microbes, Intestinal mucosal immune, BCR, CDR3 repertoire, Next generation sequencing

Posted Date: February 10th, 2021

DOI: https://doi.org/10.21203/rs.3.rs-203289/v1

License: (c) (i) This work is licensed under a Creative Commons Attribution 4.0 International License. Read Full License 


\section{Abstract \\ Background}

Colonization of gut microorganism is related to maturation of B cells in peripheral immune organs. This study aims to investigate the effect of intestinal microflora in Germ-free (GF), Specific Pathogen-free (SPF) and Clean (CL) BALB/C mice to small intestine total B-cell and memory B-cell receptor (BCR) complementary-determining region 3 (CDR3) repertoire.

\section{Results}

The composition and characteristics of intestinal microflora were analyzed by $16 \mathrm{~S}$ rDNA sequencing. Genomic DNA extracted from small intestine tissue and memory B-cells of GF, SPF and CL mice were conducted via high-throughput DNA sequencing methods. As expected, significant differences of gut microflora diversity were observed in the three mice groups. CL group showed the most diversity, followed by SPF group, and GF group had the lowest diversity. Moreover, anormogenesis of intestinal lymphoid tissue were observed in GF mice. Diversity of the BCR heavy chain CDR3 repertoire in memory B cells were significant difference among three groups, but not in total B cells. The nucleotide polymorphism, usage frequency of gene segments ( $V, D, J, V-J$ gene segments) and amino acid of total B cells and memory B cells CDR3 were comparable among three mice groups, and there was significant difference between $\mathrm{CL}$ and GF mice groups.

\section{Conclusions}

The results of this study advocate that the colonization of intestinal microorganisms affect the diversity of B cells CDR3 repertoire. Elucidating mechanism of microbiome participated in the function of intestinal mucosal immune system may have positive effects on human health, and it requires further investigation.

\section{Background}

Dysregulation of intestinal mucosal immune system can progressively lead to autoimmune disease and inflammation-driven carcinogenesis [1]. The gut microbiota has been shown to play critical roles in maintaining intestinal mucosal immune system homeostasis [2-4]. The intestinal mucosal immune system includes three different mucosal lymphoid structures: the epithelia, Peyer's patches and the lamina propria [5]. Both Peyer's patches and the lamina propria contribute to generating B cells and plasma cells. Activated B cells consistently generate IgA-producing plasma cells for T celldependent and T cell-independent responses in the gut to protecting the gut barrier [6-8]. Intestinal microorganisms can stimulate B cells to secrete SIgA, which in turn regulates the diversity of intestinal microorganisms [9]. Moreover, the number of plasma cells in GF mice is greatly reduced, but the mature IgA production can be induced by transplanting Filamenous, Clostridium or Alcaligenes to GF mice [10]. Recent studies have shown that the colonization of intestinal microorganisms can promote the maturation of B cells in peripheral immune organs [11]. However, the effects of colonization of diverse intestinal microorganisms on the intestinal total B cells and memory B cells are unknown.

$B$ cells are the main cell group that mediates the humoral immune response. Specific recognition and binding of an antigen are the BCRs expressed on the surface of B cells. Complementarity-determining region 3 (CDR3) is the most hyper-variable region in B cell receptor (BCR) gene, and the most critical structure in antigen recognition $[12,13]$. Comparing the diversity of BCR CDR3 repertoire, length distribution, IGHV, IGHD and IGHJ gene family expression and IGHV-IGHJ family distribution, the physiological and pathological state of the immune system can be evaluated [14, 15].

Germ free (GF), gnotobiotic (GN), specific pathogen free (SPF) and clean (CL) mice are conventional experimental model animals for studying the establishment and breaking of balance between intestinal microflora and intestinal immune system. Compared with specific pathogen free (SPF) animals, GF animals produce fewer IELs [16] and have significantly reduced plasma cells and Tregs in the mucosal lymphoid structures [17, 18]. In the present study, the diversity of intestinal microorganisms was assessed by $16 \mathrm{~S}$ rDNA sequencing in GF, SPF and CL mice, and BCR CDR3 repertoire diversity of total $B$ cells and memory B cells in small intestine were analyzed by high-throughput DNA sequencing methods. The mechanism of interaction between intestinal microflora and intestinal immune system was explored from the perspective of intestinal microflora and BCR heavy chain CDR3 repertoire diversity. The present study enhances the current understanding of the balance between gut microflora and intestinal mucosa immune system.

\section{Results}

\section{Morphological Differences of Intestinal Tissues and Organs}

Compared with CL and SPF grade mice, the intestinal of GF mice have characteristic changes, which are mainly manifested as the enlargement of cecum and the thin intestinal wall (Figure 1A). Moreover, significant differences in the morphology of the small intestine and mesenteric lymph nodes of GF grade mice were observed by H\&E staining sections (Figure 1B). In GF mice, the density of small intestinal villi was thinner, the length of small intestinal villi was longer, the depth of crypt was deeper, and the glandular layer and basal layer were thinner. In addition, the original germinal center of 
the mesenteric lymph nodes became smaller, and the diffuse lymphoid tissue between the superficial cortical lymph nodes under the capsule of the lymph nodes was less. It is suggested that the tissue structure of small intestine and lymph nodes of GF mice are dysplasia.

\section{Sequencing of Intestinal Flora}

In order to explore the factors that affect the intestinal mucosal immune system of three different feeding levels mice, we detected the diversity of intestinal microorganisms in GF, SPF and CL mice. The Operational Taxonomic Units (OTU) statistical results of mouse intestinal flora were shown in Additional file 1. The OTU number of intestinal flora in GF, SPF and CL groups were about 50, 280 and 330, respectively. The results of principal component analysis showed that the composition of intestinal microflora was different in three grades of mice, and the composition of intestinal microflora in SPF group and CL group were similar (Additional file 2). OTU Rank analysis showed that the species richness and evenness of intestinal microflora were the lowest in GF mice, and the diversity of intestinal microflora was relatively rich in SPF and CL mice, among which CL mice were the most abundant (Additional file 2).

\section{Species Composition and Clustering of Intestinal Flora}

By comparing the sequencing results of intestinal flora with the available database, the features of species classification of intestinal flora were conducted (Figure 2 and Additional file 3). About $99 \%$ of the fecal flora of GF grade mice were Brevibacilli in the phylum Firmicutes. The intestinal microflora of SPF and CL mice were mainly composed of Firmicutes and Bacteroidetes, but the proportion of the two groups were different. Bacteroides was the main composition of intestinal microflora in SPF mice, mainly including s24-7, rikenellaceae, prevotellaceae, bacteroideae and paraprevotellaceaes. The intestinal microflora of CL mice was mainly composed of Firmicutes, including clostridia under clostridia. Further analysis showed that about $10 \%$ of the unique intestinal microflora in $\mathrm{CL}$ group were desulfovibrionaceae and helicobacteraceae under proteobacteria. Moreover囚the cluster analysis of six classification levels of intestinal microorganisms also indicated that the species of intestinal microorganisms in GF group were less, and almost all of them were Bacillus brevis.

\section{Species Diversity Analysis of Intestinal Flora}

The Chao index, Shannon index, Ace index, Simpson index and Observed species index were used to evaluate the alpha diversity of the samples, and the results showed that there were significant differences in the five diversity indexes of intestinal flora among the three groups $(P<0.05)$, suggesting that the diversity of intestinal flora were different among the three groups. The diversity of intestinal flora was the most abundant in $\mathrm{CL}$ mice, followed by SPF mice, and the lowest in GF mice (Additional file 4).

\section{Detection of BCR Heavy Chain CDR3 Sequence}

High-throughput sequencing was performed on memory B cells and total B cells in small intestine of GF, SPF and CL mice to obtain BCR heavy chains CDR3 libraries. Each library was filtered to obtain high-quality reads and compared with databases. The statistical results were shown in Table 1 . The ratio of unique nucleotides to total nucleotides and the ratio of productive unique nucleotides to total productive nucleotide in $B$ cells $B C R$ CDR3 sequence were lower than those in memory $B$ cells in all three groups. The ratio of unique CDR3 amino acids to total CDR3 amino acids and the ratio of unique in frame CDR3 amino acids to total in frame CDR3 amino acids were higher than those in memory B cells in GF and SPF groups, and the CL group was the opposite.

\section{Diversity of the BCR Heavy Chain CDR3 Repertoire}

The inverse Simpson's diversity index (1/DS) for small intestine BCR heavy-chain CDR3 repertoire of total and memory B cells were showed in Figure 3. The values of 1/DS were significant in different animal groups among total and memory B cells. The highest index of total B cells was detected in SPF group, followed by GF group, and the lowest was CL group. For memory B cells, the 1/DS index was positively correlated with the feeding level of mice.

\section{Usage Frequency of V, D and J Gene Segments of the BCR Heavy Chain CDR3 Region}

The usage frequency of the V, D and J gene segment in the BCR Heavy chain CDR3 were determined among three groups. IGHV03-02 IGHV14-02

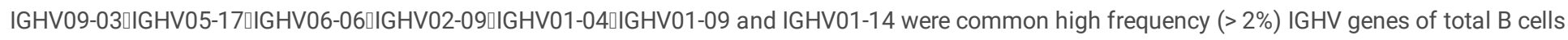
in all groups. IGHV14-02 IGHV03-02 and IGHV09-03 were common high frequency IGHV genes of memory B cells in all groups. Holm-Sidak Test showed that the distribution of the IGHV gene found in total B cells and memory B cells were significant difference among three groups. In addition, some IGHV genes disappeared in GF and CL groups, for instance, IGHV08-04 and IGHV02-02-1 genes of total B cells, only found in GF and SPF groups (Additional file 5).

Similarly, common high-frequency IGHD genes were found in the total B cells (Figure 4 A) and memory B cells (Figure 4 B) of the three groups of mice. However, both in total B cells and memory B cells, the difference of IGHD genes distribution among three groups were insignificant $(P>0.05)$.

IGHJ04-01 and IGHJ03-01 were common high-frequency IGHJ genes both in total B cells (Figure 4 C) and memory B cells (Figure 4 D) in three groups. Statistical analysis showed that the usage frequency of IGHJ04-01 gene in BCR heavy chain CDR3 repertoire of total B cells of small intestine in CL group was significantly lower than that in GF and SPF group $(P<0.05)$, while the usage frequency of IGHJ04-01 gene of memory B cells in GF groups was the lowest and did differ significantly between GF and SPF groups in terms of IGHJO4-01 gene distribution. 
Hierarchical clustering heat map was created to comparatively analyze the combinations of total B cells (Figure 4 E) and memory B cells (Figure 4 F) $\mathrm{BCR}$ heavy chain $\mathrm{V}$ and $\mathrm{J}$ genes in the three groups, and T-test was performed to examine the distribution ratio of combinations. Five (IGHVO6-06-

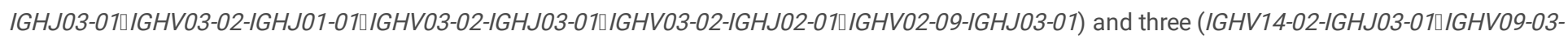
IGHJ04-01 IGHV09-03-IGHJ02-01)common combinations were found in total B cells and memory B cells of all groups. The combinations in total B cells between GF group and SPF group was relatively close, while SPF and CL group was relatively close in memory B cells. Compared with GF groups, one combination (IGHV03-02-IGHJ03-01) of total B cells and two combinations (IGHV03-02-IGHJ03-01and IGHV14-02-IGHJ04-01) of memory B cells were significantly up-regulated both in $\mathrm{CL}$ and SPF groups. In addition, the combinations (IGHV01-80-IGHJ04-01) of total B cells was significantly down-regulated both in GF and SPF groups by comparing with CL groups.

\section{Amino Acid Sequences in the BCR Heavy Chain CDR3 Repertoire}

In analysis of amino acid sequences, Tyrosine was found as most popular amino acid in the BCR heavy chain CDR3 repertoire of total (Figure 5 A) and memory B cells (Figure 5 B) in all three groups, the usage frequency was about $20 \%$. Statistical analysis showed that the usage frequency of methionine was significantly decreased in total B cells of both CL and SPF groups by comparing to GF group, while was significantly increased in memory B cells of SPF groups. The average CDR3 length of nine BALB/c mice were 12 amino acid-based Gaussian distribution, and statistical analysis showed that the difference in the CDR3 length distribution of total (Figure $5 \mathrm{C}$ ) and memory B cells (Figure 5 D) among the three groups was insignificant $(P>0.05)$.

The overlap amino acid sequence was also analyzed both in total B cells (Figure $5 \mathrm{E}$ ) and memory B cells (Figure $5 \mathrm{~F}$ ). 37 amino acid sequences were shared in the total B cells of the nine BALB/C mice, and only one amino acid sequence was shared in the memory B cells of the nine BALB/C mice. However, the frequency of all the common amino acid sequences was low (<0.5\%). Top 10 most frequent amino acid sequences in each mouse total $B$ cells and memory B cells were also detected (data not shown), the highly conserved amino acid motif "WYFDV " was found in all three group's total B cells, and the most was found in GF group. Two highly conserved amino acid motifs ("RYFDV" and "WYFDV") were detected in all three group's memory B cells. Interestingly, the most was also found in GF groups.

\section{Mutations Frequency of BCR Heavy Chain CDR3 Repertoire}

The statistical results of nucleotide polymorphism of CDR3 repertoire of total B cells and memory B cells in three experimental groups were shown in Figure 6. The insertion and deletion of nucleotides in total B cells were significantly different in three experimental groups $(P<0.05)$, including the number of total nucleotide insertion, D5 'deletion, J gene deletion, V-D gene insertion and D-J gene insertion. In contrast, amino acid insertion and deletion in memory B cells were significantly different only at the D3 ' deletion $(P<0.05)$.

Further analysis of high frequency mutation of $\mathrm{V}$ gene showed that the proportion of $\mathrm{V}$ gene mutation in total $\mathrm{B}$ cells was different in three groups of experimental animals, and the proportion of mutation sequence in CL was significantly higher than that in SPF and GF groups with $400-800$ mutations per 10,000 bases. The proportion of mutation sequences in memory B cells was about $70 \%$, and there was no significant difference among the three experimental groups.

\section{Discussion}

BALB / c mice have been widely used in immunology research because of their small individual differences, pure genetic genes and good overall quality. However, different feeding environment may affect intestinal immunity[11]. The characteristics of immune organs and tissues in GF experimental animals were described as follows: thymic epithelial cells became larger, cytoplasmic vesicular structure and lysosome became smaller; the development of spleen and peripheral lymph nodes were limited; cecum became larger, intestinal wall became thinner, mesenteric lymph nodes and Pais lymph nodes were smaller, and primary germinal center became smaller[25-27]. In this study, the histological morphology of small intestine and lymph nodes of GF mice was significantly different from that of CL and SPF, which was consistent with the early literature, suggesting that different the feeding environment may affect the mucosal immune system of mice.

Intestinal dysfunction is related to the changes in intestinal microbes[28]. Uncovering the composition of the microbiota and its metabolic products can help determine novel biomarkers of the disease and help identify new therapeutic targets. In this study, the intestinal microflora of GF, SPF and CL mice were different. The species of the intestinal microflora of GF mice were almost Brevibacillus, and the species composition of the intestinal microflora of SPF and CL mice were mainly composed of Firmicutes and Bacteroidetes, which was consistent with the previous literature[29, 30]. Moreover, qualitative and quantitative analysis showed that there were significant differences in species richness, evenness and diversity of intestinal flora among the three groups. Among them, $\mathrm{CL}$ group had the richest species diversity and evenness, followed by SPF group, while GF group had the lowest. The composition of intestinal microflora among different feeding levels of mice were also different. The higher the feeding level, the less the species composition of intestinal microflora.

The colonization of intestinal microorganisms may affect the diversity of the immune repertoire. The diversity of IgA repertoire in the small intestine of GN mice is more than that of GF mice[31], and Chen et al. demonstrated that microbial symbionts influence host immunity by enriching frequencies of 
antibacterial specificities within preimmune B cell repertoires[32]. As the BCR diversity plays an essential role in effective immune response, the high diversity in subtypes of immune proteins is expected to have more effective immune response against pathogens [33]. Here, we analyzed the BCR heavy chain CDR3 repertoire diversity of intestinal memory B cells and total B cells in GF mice, SPF mice and CL mice. Compared with GF group, the diversity of BCR heavy chain CDR3 repertoire of small intestine memory B cells in SPF group and CL group was more abundant, but the diversity of $B C R$ heavy chain CDR3 repertoire of total $B$ cells was not significant. It is suggested that the diversity of intestinal flora may be related to the diversity of BCR heavy chain CDR3 repertoire of small intestinal memory B cells in BALB / c mice.

During the development of B lymphocytes, $\mathrm{V}, \mathrm{D}$, and $\mathrm{J}$ gene segments of BCR are rearranged. It has been found that the expression of $\mathrm{V}$ gene was significant different in gut lamina propria B cells of GF and GN mice[32, 34]. In this study, IGHV03-02-IGHV14-02-IGHV09-03ロIGHD01-010IGHJ04-010 IGHJ03-01 and IGHV09-03-IGHJ02-01 combination are common high-frequency genes both in total and memory B cells of all three groups. In T test for frequency usage of BCR heavy chain V, D, J, and V-J segments, we identified two up-regulated genes in total B cells: IGHV03-06 and V-J combination (IGHV03-02- IGHJ03-01); and two down-regulated genes: IGHV01-09 and IGHV15-02 by comparing with GF groups. These abnormal expressions of BCR heavy chain CDR3 of V, D, J, and V-J combinations sub-family gene may be involved in the development disorder of immune organs in intestinal mucosa of GF mice. Moreover, these up-regulated genes in CL and SPF mice may be associated with BCR-specific clonal proliferation of B lymphocytes.

During BCR germline gene recombines, different number of nucleotides can either randomly insert into V-J segments or V-D-J segments. Oppositely, different number of nucleotides can randomly delete in V-J segments or V-D-J segments. Through the insertion or deletion, a highly diversity variable CDR3 region can be generated in ways of differences in length and amino acid sequences of the BCR CDR3[35, 36]. In this study, there were different numbers of inserted and deleted nucleotides in BCR heavy chain CDR3 region of memory B cells and total B cells of three groups of mice. Compared with SPF group, the number of inserted and deleted nucleotides was significantly increased, which may affect the length distribution of amino acid sequence in CDR3 region.

In addition, the $\mathrm{V}$ gene is considered to be a high frequency mutation region. It has been reported that the increase in the number of $\mathrm{V}$ gene mutations is positively related to the affinity of antigen binding[37, 38]. The proportion of mutation in $\mathrm{V}$ gene region of total $\mathrm{B}$ cells in $\mathrm{CL}$ group was significantly higher than that in SPF and GF groups, which may be related to the abundance of small intestinal flora in CL group.

\section{Conclusion}

We analyzed the diversity of intestinal microflora in GF, SPF and CL mice. The colonization of intestinal microflora may affect the development and maturation of the small intestine and lymph nodes. We constructed BCR heavy chain libraries of total B cells and memory B cells in the small intestine of GF, SPF and CL mice, and found that the diversity of total B cells and memory B cells CDR3 repertoire in GF, SPF and CL mice was different, suggesting that the colonization of intestinal microflora may affect the immune response and tolerance of $B$ cells.

\section{Materials And Methods}

\section{Sample Collection}

A total of nine female BALB/c mice were purchased from the Department of experimental zoology, the basic Department of the China Third Military Medical University. Among them, there were three GF mice, SPF mice and CL mice respectively, each weighing $21-24 \mathrm{~g}$. All animals and experiments were conducted according to the guidance of animal care and use of laboratory animals (Ministry of health, China) and approved by the experimental animal and use ethics committee of Zunyi Medical University. Feces of all mice were collected and sent to Beijing Genomics institution for sequencing of intestinal microorganisms. Small intestine and mesenteric lymph nodes were collected at the time of sacrifice by cervical dislocation.

\section{Morphological Analysis}

The small intestine and mesenteric lymph nodes were fixed in $4 \%$ paraformaldehyde overnight at $4{ }^{\circ} \mathrm{C}$, embedded in paraffin, sectioned into 8 - $\mu \mathrm{m}$ thick slices, deparaffinized, and stained with hematoxylin and eosin (H\&E). Tissue morphology was observed under a light microscope.

\section{Sequencing of Intestinal Microorganisms}

Fecal DNA was extracted as described previously[19]. 16S rDNA obtained from the fecal extraction was analyzed by Illumina sequencing according to the 16S Metagenomic Sequencing Library Preparation (15044223B) protocol. The experimental method of 16S rDNA sequencing was described by Watanabe et al. [20]. The DNA library was sequenced using MiSeq Reagent Kit V3(Illumina Inc.) in the MiSeq platform according to the manufacturer's instructions. The 16S rDNA sequences were analyzed by the Quantitative Insights into Microbial Ecology (QIIME) pipeline (version 1.9.1) [21]. Through quality control, data filter out low-quality sequences, splice the sequences into tags through similarity relationship between sequences, gather tags into operational taxonomic units (OTU) based on whether they had $97 \%$ homology with the UCLUST algorithm, and then compare OTU with database (August 2013 version) to annotate species of OTU. Taxonomy from the phylum to the genus level was also performed by using the QIIME pipeline to analysis these sequences.

\section{Memory B Cells Isolation and DNA Extraction}


Single cell suspensions were prepared by grinding the tissues with the plunger of a $5 \mathrm{~mL}$ disposable syringe. Memory B cells were isolated using the Memory B Cell Isolation Kit (Miltenyi Biotec.) according to the manufacturer's instructions. Cells were fluorescently stained with CD45R (B220)-VioBlue (\#130-094-287), CD38-PE (\#130-097-087) and Anti-IgM-FITC (\#130-095-906) and analyzed by flow cytometry using the MACSQuant ${ }^{\circledR}$ Analyzer. Cell debris and dead cells were excluded from the analysis based on scatter signals and propidium iodide fluorescence. Genomic DNA was extracted from isolated memory B cells and small intestine tissue using the QIAamp DNA MiniKit (Qiagen, Milano, Italy) according to the manufacture's instruction.

\section{BCR heavy ChainRepertoire Preparation and High-Throughput Sequencing}

The concentration and purity of genomic DNA of samples were conformed for BCR heavy chain CDR3 sequencing (http://www.immunoseq.com). And then genomic DNA were sent to Adaptive Biotechnologies Corp (Seattle, WA, US) for sequencing. BCR heavy chain CDR3 sequences were generated as described previously [22]. Briefly, a multiplex polymerase chain reaction (PCR) amplification was performed consisting of upstream and downstream primers that targeted all possible somatic combinations of the rearranged BCR heavy chain CDR3. The 5' end of each primer had a unique MID sequence consisting of 10 nucleotides. The PCR reaction system and conditions were described previously [23]. PCR products were recovered using agarose gel purification kit (Qiagen) in accordance with the manufacturer's protocol. After quantitation using capillary electrophoresis, libraries were pooled accordingly and sequenced.

\section{Bioinformatics Analysis of BCR Heavy Chain Library}

The IMGT website (http://www.imgt.org/) was used to build a reference sequence dataset. Raw sequences in the FASTA format were processed by Immuno-SEQ analyzer toolset and IMGT/High V-QUEST (version 1.3.1) to remove the No results and Un-known sequences as well as out of frame sequences. Using Immuno-SEQ analyzer toolset and IMGT/High V-QUEST, the characteristics of the BCR heavy chain CDR3 repertoire sequences were defined, including CDR3 nucleotide; CDR3 amino acid; count (reads); frequency count (\%); CDR3 length; V gene name; D gene name; J gene name; $V$ deletion; $\mathrm{n} 1$ insertion; D 5' deletion; D 3' deletion; $\mathrm{n} 2$ insertion; J deletion; V index; n1 index; D index; $\mathrm{n} 2$ index; J index; sequence status (Has stop/in frame/out frame). The V-J rearrangement of the CDR3 repertoire, the proportion and frequency of unique CDR3 sequences, CDR3 repertoire clonality, CDR3 amino acid length, CDR3 animo acid usage, $\mathrm{V}$ deletion and $\mathrm{J}$ deletion, and dominant $\mathrm{V}-\mathrm{J}$ combination gene segments were also calculated in different mouse groups and different B cells (naïve and memory) samples. Additionally, R package "ggplot2", "Venn Diagram", and GraphPad Prism (version 5) were used to plot the figures. Data analysis was performed by R studio (v3.3.3) and GraphPad Prism (version 5) software. P-values were calculated with the aid of the $t$ test. $P<0.05$ was considered statistically significant.

The OTU rank curve was used to explain the richness and evenness of species in intestinal flora. The wider the curve is, the more uniform the composition of intestinal flora is. Besides, the Chao index, Shannon index, Ace index, Simpson index and Observed species index were used to evaluate the alpha diversity of intestinal microflora. The larger the first four indexes and the smaller the last index, the more abundant the species in the samples. In order to count the clone frequency of CDR3 region, the inverse Simpson's diversity index (1/DS) was performed. The correction formula of 1/DS as below:, which ni represents the total number of the i-th sequence, and the higher the 1 / DS value, the richer the diversity [24].

\section{Abbreviations}

Germ-free: GF; Specific Pathogen-free: SPF; Clean: CL; B-cell receptor: BCR; complementary-determining region 3: CDR3; OUT: Operational Taxonomic Units; PCR: polymerase chain reaction; IMGT: International ImMunoGeneTics.

\section{Declarations}

\section{Funding}

The work was supported by grants from the National Natural Science Foundation of China (81860300\&81660269) and Guizhou Provincial High-level Innovative Talents Project (No. [2018] 5637).

\section{Author contributions}

$\mathrm{XY}$ designed the research, JL, YP and QM did the experiment and wrote the paper. XH, LM, BS and XW analyzed parts of the data. All authors contributed to the article and approved the submitted version.

\section{Conflict of interest}

The authors declare that the research was conducted in the absence of any commercial or financial relationships that could be construed as a potential conflict of interest.

\section{Availability of data and materials}

The original data sets generated and analyzed during this study are made available by the corresponding author upon reasonable request.

Ethics approval and consent to participate 
All animals and experiments were conducted according to the guidance of animal care and use of laboratory animals (Ministry of health, China) and approved by the experimental animal and use ethics committee of Zunyi Medical University.

Acknowledgements

We would like to express our gratitude to all those who helped us during the data analysis and manuscript writing.

Consent for publication

Not applicable.

\section{References}

1. Pickard JM, Zeng MY, Caruso R, Nunez G: Gut microbiota: Role in pathogen colonization, immune responses, and inflammatory disease. Immunol Rev 2017, 279(1):70-89.

2. Dey P: Targeting Gut Barrier Dysfunction With Phytotherapies: Effective Strategy Against Chronic Diseases. Pharmacol Res 2020.

3. Zheng Y, Gou X, Zhang L, Gao H, Wei Y, Yu X, Pang B, Tian J, Tong X, Li M: Interactions Between Gut Microbiota, Host, and Herbal Medicines: A Review of New Insights Into the Pathogenesis and Treatment of Type 2 Diabetes. Front Cell Infect Microbiol 2020, 10:360.

4. Shi N, Li N, Duan X, Niu HJMMR: Interaction between the gut microbiome and mucosal immune system. $2017,4: 14$.

5. Mowat A, Agace WJNrl: Regional specialization within the intestinal immune system. 2014, 14(10):667-685.

6. Bemark M, Boysen P, Lycke NY: Induction of gut IgA production through T cell-dependent and T cell-independent pathways. Ann N Y Acad Sci 2012, 1247:97-116.

7. Bergqvist P, Stensson A, Lycke NY, Bemark M: T cell-independent IgA class switch recombination is restricted to the GALT and occurs prior to manifest germinal center formation. J Immunol 2010, 184(7):3545-3553.

8. Carasi P, Racedo SM, Jacquot C, Romanin DE, Serradell MA, Urdaci MC: Impact of kefir derived Lactobacillus kefiri on the mucosal immune response and gut microbiota. J Immunol Res 2015, 2015:361604.

9. Macpherson AJ, Uhr T: Induction of protective IgA by intestinal dendritic cells carrying commensal bacteria. Science 2004, 303(5664):1662-1665.

10. Umesaki Y, Setoyama H: Structure of the intestinal flora responsible for development of the gut immune system in a rodent model. Microbes Infect 2000, 2(11):1343-1351.

11. Beura LK, Hamilton SE, Bi K, Schenkel JM, Odumade OA, Casey KA, Thompson EA, Fraser KA, Rosato PC, Filali-Mouhim A et al: Normalizing the environment recapitulates adult human immune traits in laboratory mice. Nature 2016, 532(7600):512-516.

12. Dondelinger M, Filee P, Sauvage E, Quinting B, Muyldermans S, Galleni M, Vandevenne MS: Understanding the Significance and Implications of Antibody Numbering and Antigen-Binding Surface/Residue Definition. Front Immuno/ 2018, 9:2278.

13. Henry KA, MacKenzie CR: Antigen recognition by single-domain antibodies: structural latitudes and constraints. MAbs 2018, 10(6):815-826.

14. Liu S, Zhu Y, Lin LW, Ding SK, Lin XC, Zhong KL, Pan K, Dai Y: The composition and variation of the BCR CDR3s in gastric cancer. Oncol Lett 2018, 16(1):239-246.

15. Shi X, Shao T, Huo F, Zheng C, Li W, Jiang Z: An analysis of abnormalities in the B cell receptor repertoire in patients with systemic sclerosis using high-throughput sequencing. PeerJ 2020, 8:e8370.

16. Bandeira A, Mota-Santos T, Itohara S, Degermann S, Heusser C, Tonegawa S, Coutinho A: Localization of gamma/delta T cells to the intestinal epithelium is independent of normal microbial colonization. $J$ Exp Med 1990, 172(1):239-244.

17. Ostman S, Rask C, Wold AE, Hultkrantz S, Telemo E: Impaired regulatory T cell function in germ-free mice. Eur J Immuno/ 2006, 36(9):2336-2346.

18. Shi N, Li N, Duan X, Niu H: Interaction between the gut microbiome and mucosal immune system. Mil Med Res 2017, 4:14.

19. Nishijima S, Suda W, Oshima K, Kim SW, Hirose Y, Morita H, Hattori M: The gut microbiome of healthy Japanese and its microbial and functional uniqueness. DNA Res 2016, 23(2):125-133.

20. Watanabe A, Sasaki H, Miyakawa H, Nakayama Y, Lyu Y, Shibata S: Effect of Dose and Timing of Burdock (Arctium lappa) Root Intake on Intestinal Microbiota of Mice. Microorganisms 2020, 8(2).

21. Caporaso JG, Kuczynski J, Stombaugh J, Bittinger K, Bushman FD, Costello EK, Fierer N, Pena AG, Goodrich JK, Gordon JI et al: QIIME allows analysis of high-throughput community sequencing data. Nat Methods 2010, 7(5):335-336.

22. Yan Q, Wang L, Lai L, Liu S, Chen H, Zhang J, Dai Y, Sui W: Next generation sequencing reveals novel alterations in B-cell heavy chain receptor repertoires associated with acute-on-chronic liver failure. Int J Mol Med 2019, 43(1):243-255.

23. Chen D, Zhang Z, Yang Y, Hong Q, Li W, Zhuo L: High-throughput sequencing analysis of genes encoding the B-lymphocyte receptor heavy-chain CDR3 in renal and peripheral blood of IgA nephropathy. Biosci Rep 2019, 39(10).

24. Wu J, Liu D, Tu W, Song W, Zhao X: T-cell receptor diversity is selectively skewed in T-cell populations of patients with Wiskott-Aldrich syndrome. J Allergy Clin Immunol 2015, 135(1):209-216.

25. Mebius RE: Organogenesis of lymphoid tissues. Nat Rev Immunol 2003, 3(4):292-303.

Page $7 / 12$ 
26. Abrams GD, Bauer H, Sprinz H: Influence of the normal flora on mucosal morphology and cellular renewal in the ileum. A comparison of germ-free and conventional mice. Lab Invest 1963, 12:355-364.

27. Sommer F, Backhed F: The gut microbiota-masters of host development and physiology. Nat Rev Microbio/ 2013, 11(4):227-238.

28. Villapol S: Gastrointestinal symptoms associated with COVID-19: impact on the gut microbiome. Trans/ Res 2020.

29. Qian LJ, Kang SM, Xie JL, Huang L, Wen Q, Fan YY, Lu LJ, Jiang L: Early-life gut microbial colonization shapes Th1/Th2 balance in asthma model in BALB/c mice. BMC Microbiol 2017, 17(1):135.

30. Wang Y, Sun L, Chen S, Guo S, Yue T, Hou Q, Feng M, Xu H, Liu Y, Wang P et al: The administration of Escherichia coli Nissle 1917 ameliorates irinotecan-induced intestinal barrier dysfunction and gut microbial dysbiosis in mice. Life Sci 2019, 231:116529.

31. Lindner C, Thomsen I, Wahl B, Ugur M, Sethi MK, Friedrichsen M, Smoczek A, Ott S, Baumann U, Suerbaum S et al: Diversification of memory B cells drives the continuous adaptation of secretory antibodies to gut microbiota. Nat Immunol 2015, 16(8):880-888.

32. Chen Y, Chaudhary N, Yang N, Granato A, Turner JA, Howard SL, Devereaux C, Zuo T, Shrestha A, Goel RR et al: Microbial symbionts regulate the primary Ig repertoire. J Exp Med 2018, 215(5):1397-1415.

33. Hoehn KB, Fowler A, Lunter G, Pybus OG: The Diversity and Molecular Evolution of B-Cell Receptors during Infection. Mol Biol Evol 2016.

34. Wesemann DR, Portuguese AJ, Meyers RM, Gallagher MP, Cluff-Jones K, Magee JM, Panchakshari RA, Rodig SJ, Kepler TB, Alt FW: Microbial colonization influences early B-lineage development in the gut lamina propria. Nature 2013, 501(7465):112-115.

35. Thornqvist L, Ohlin M: Data on the nucleotide composition of the first codons encoding the complementary determining region 3 (CDR3) in immunoglobulin heavy chains. Data Brief 2018, 19:337-352.

36. Rettig TA, Ward C, Bye BA, Pecaut MJ, Chapes SK: Characterization of the naive murine antibody repertoire using unamplified high-throughput sequencing. PLoS One 2018, 13(1):e0190982.

37. Mishra AK, Mariuzza RA: Insights into the Structural Basis of Antibody Affinity Maturation from Next-Generation Sequencing. Front Immuno/2018, 9:117.

38. Wu YC, James LK, Vander Heiden JA, Uduman M, Durham SR, Kleinstein SH, Kipling D, Gould HJ: Influence of seasonal exposure to grass pollen on local and peripheral blood IgE repertoires in patients with allergic rhinitis. J Allergy Clin Immunol 2014, 134(3):604-612.

\section{Tables}

Table 1 Total sequences statistics of BCR heavy chain CDR3 repertoire

\begin{tabular}{|c|c|c|c|c|c|c|c|c|c|c|c|c|c|}
\hline \multirow[t]{2}{*}{ Sample } & & \multicolumn{6}{|c|}{ CDR3 Nucleotide } & \multicolumn{6}{|c|}{ CDR3 AA } \\
\hline & & $\begin{array}{l}\text { Total } \\
(\mathrm{T})\end{array}$ & $\begin{array}{l}\text { Unique } \\
\text { (U) }\end{array}$ & $\mathrm{U} / \mathrm{T}$ & $\begin{array}{l}\text { Productive } \\
\mathrm{T}(\mathrm{PT})\end{array}$ & $\begin{array}{l}\text { Productive } \\
\text { U (PU) }\end{array}$ & PU/PT & $\begin{array}{l}\text { Total } \\
(\mathrm{T})\end{array}$ & $\begin{array}{l}\text { Unique } \\
(U)\end{array}$ & $\mathrm{U} / \mathrm{T}$ & $\begin{array}{l}T \text { in } \\
\text { frame } \\
(T F)\end{array}$ & $\begin{array}{l}\text { U in } \\
\text { frame } \\
\text { (UF) }\end{array}$ & UF/TF \\
\hline \multirow{3}{*}{$\begin{array}{l}\text { Total } \\
\text { B cells }\end{array}$} & GF & 6599 & 5476 & $82.98 \%$ & 4724 & 3872 & $81.96 \%$ & 4969 & 3525 & $70.94 \%$ & 4724 & 3334 & $70.58 \%$ \\
\hline & SPF & 15771 & 12779 & $81.03 \%$ & 11539 & 9198 & $79.71 \%$ & 12129 & 7850 & $64.72 \%$ & 11539 & 7423 & $64.33 \%$ \\
\hline & $\mathrm{CL}$ & 3826 & 2728 & $71.30 \%$ & 2884 & 2003 & $69.45 \%$ & 3005 & 1401 & $46.62 \%$ & 2885 & 1329 & $46.07 \%$ \\
\hline \multirow{3}{*}{$\begin{array}{l}\text { Memory } \\
\text { B cells }\end{array}$} & GF & 260 & 250 & $96.15 \%$ & 160 & 153 & $95.63 \%$ & 173 & 93 & $53.76 \%$ & 160 & 85 & $53.13 \%$ \\
\hline & SPF & 2264 & 2145 & $94.74 \%$ & 1665 & 1578 & $94.77 \%$ & 1738 & 890 & $51.21 \%$ & 1665 & 844 & $50.69 \%$ \\
\hline & $\mathrm{CL}$ & 1410 & 1194 & $84.68 \%$ & 1002 & 850 & $84.83 \%$ & 1063 & 765 & $71.97 \%$ & 1002 & 720 & $71.86 \%$ \\
\hline
\end{tabular}

GF: Germ-free mice; SPF: Specific Pathogen-free mice; CL: Clean mice.

\section{Figures}


A

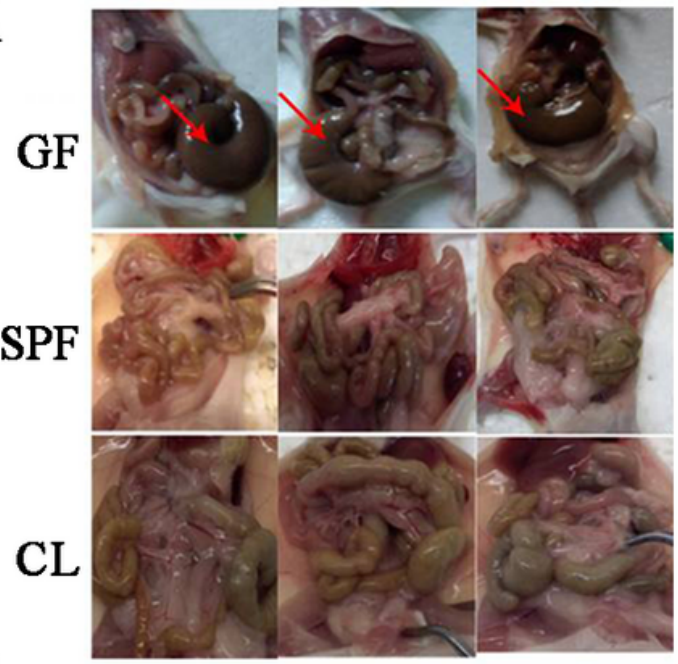

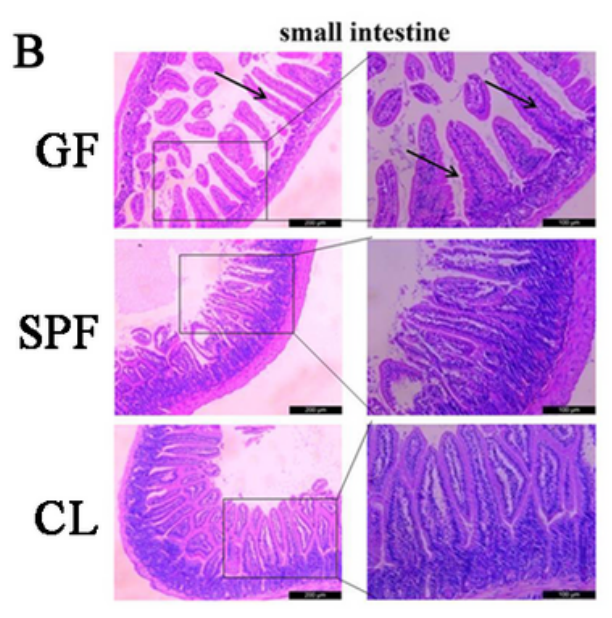

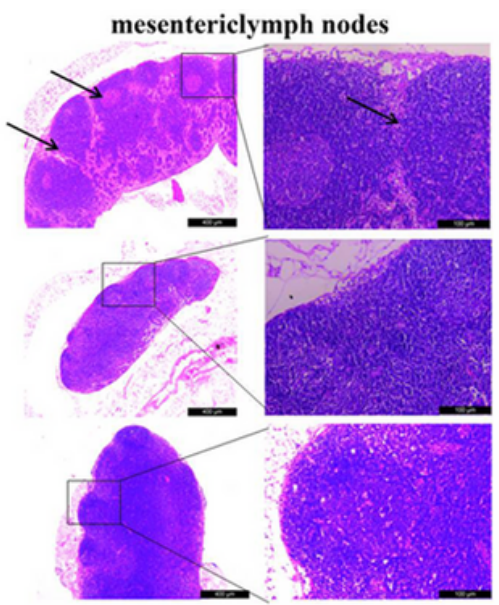

Figure 1

Morphological analysis of intestine among GF, SPF and CL BALB/c mice. A: The naked eye of intestine; B: H\&E staining of pathological sections of small intestine and mesenteric lymph nodes (40 times and 200 times). GF: Germ-free mice; SPF: Specific Pathogen-free mice; CL: Clean mice.

$\mathbf{A}_{\mathrm{R}}$
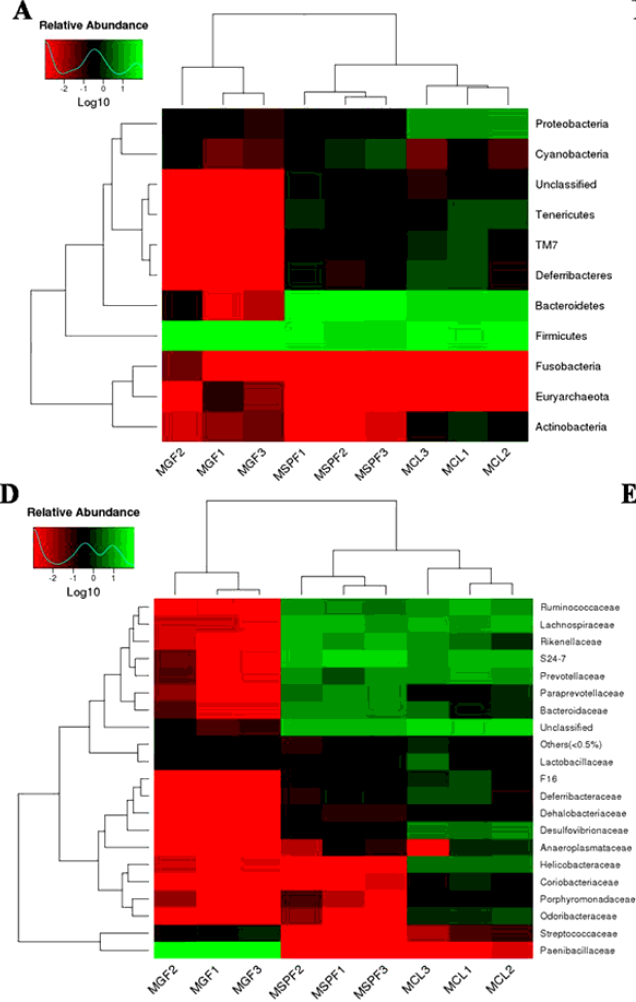

B
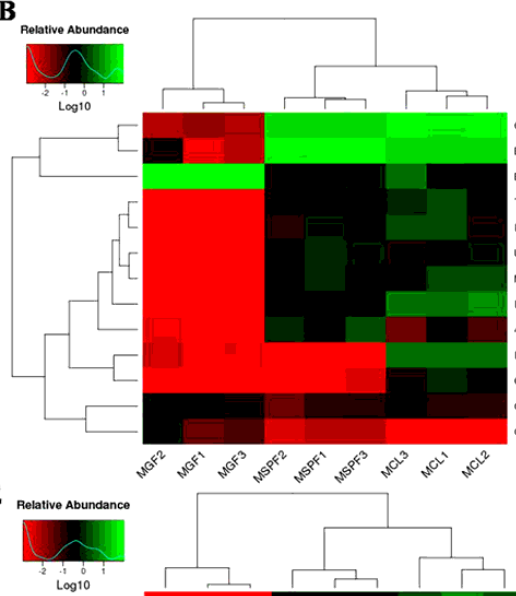

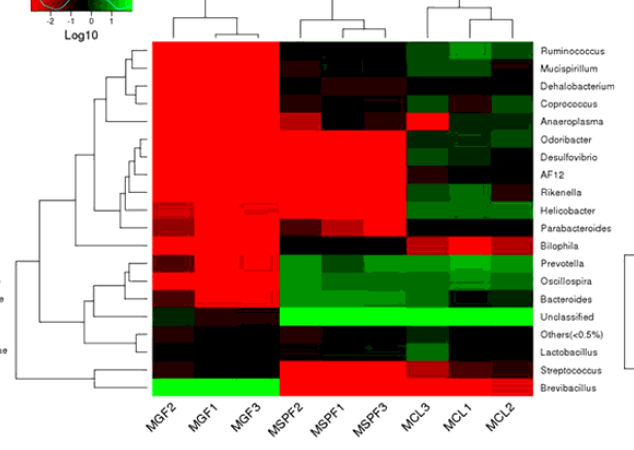

C
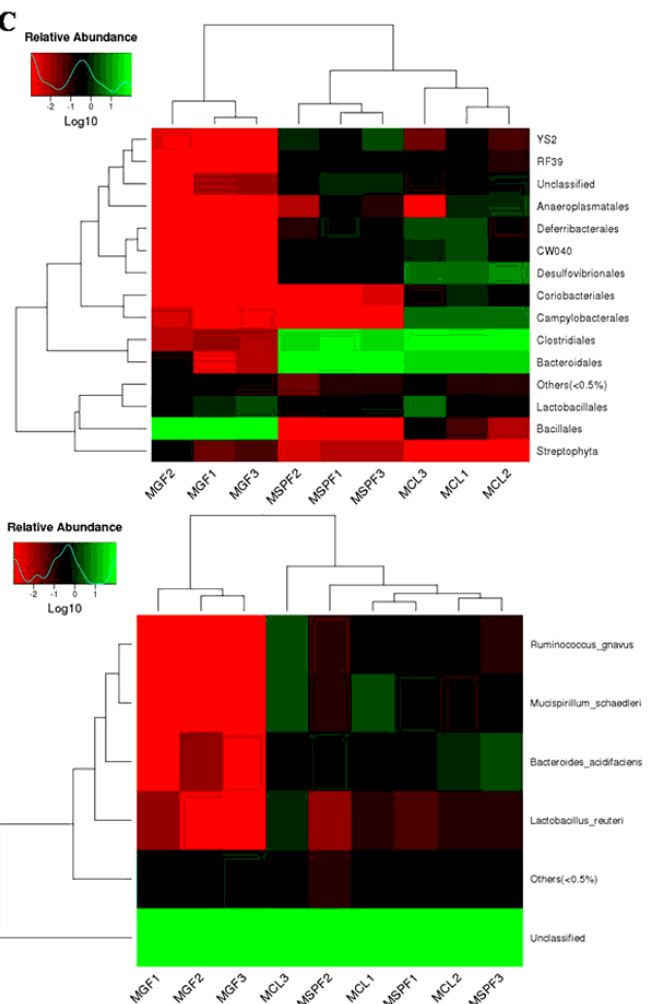

Figure 2

Species abundance thermal map of intestinal microbes as six levels (phylum, class, order, family, genus, species; A-F) of GF, SPF and CL BALB/c mice. MGF: Germ-free mice; MSPF: Specific Pathogen-free mice; MCL: Clean mice. 

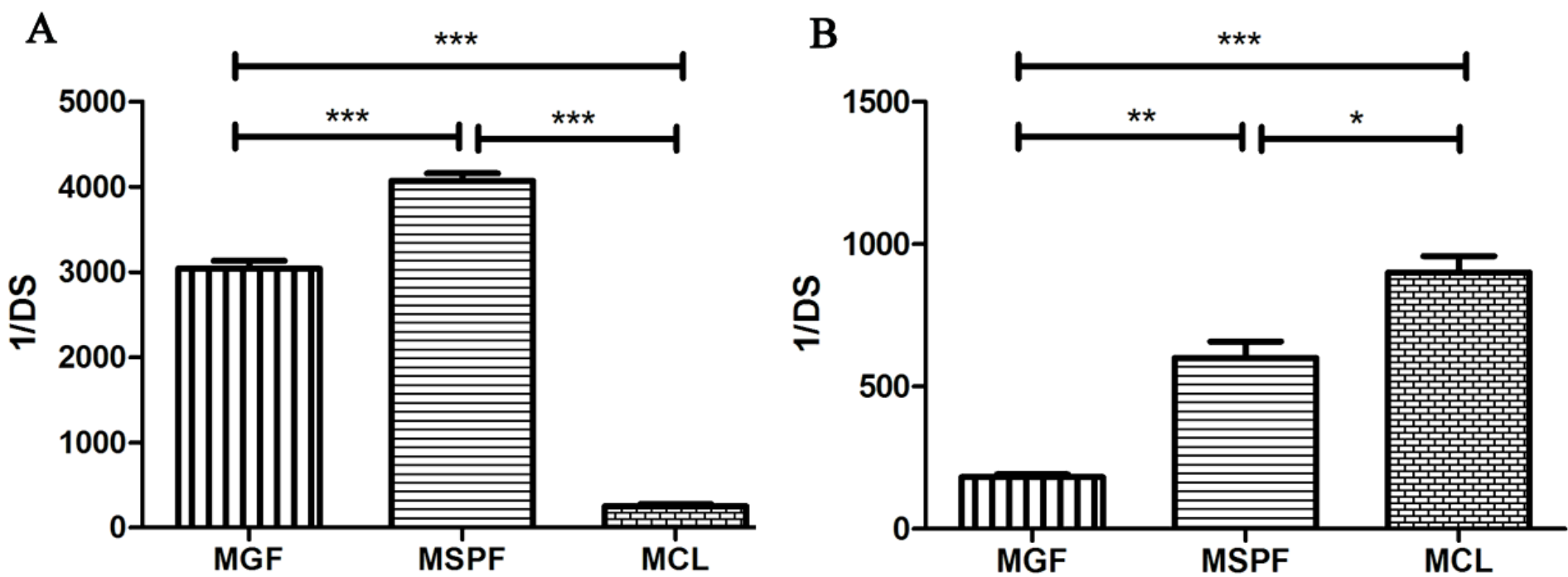

Figure 3

The anti-Simpson index statistical analysis of total and memory B cell BCR heavy chain CDR3 repertoire in small intestinal of GF, SPF and CL BALB/C mice. A: 1/DS for total B cells; B: 1/DS for memory B cells. 1/DS: the inverse Simpson's diversity index; MGF: Germ-free mice; MSPF: Specific Pathogen-free mice; MCL: Clean mice.

A

C
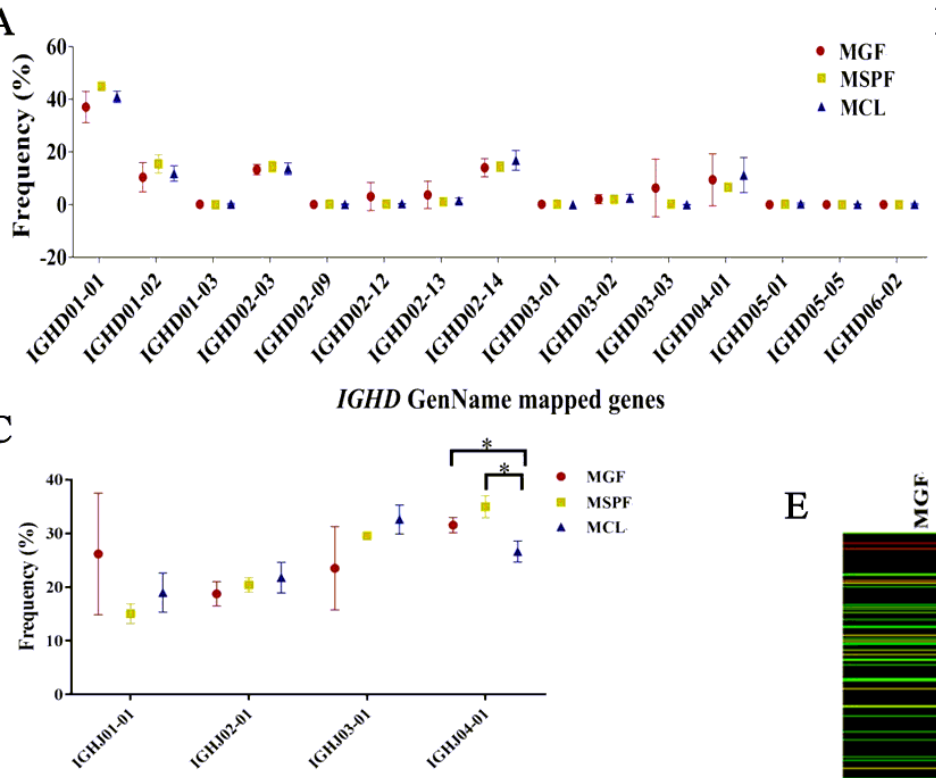

$\mathrm{D}$

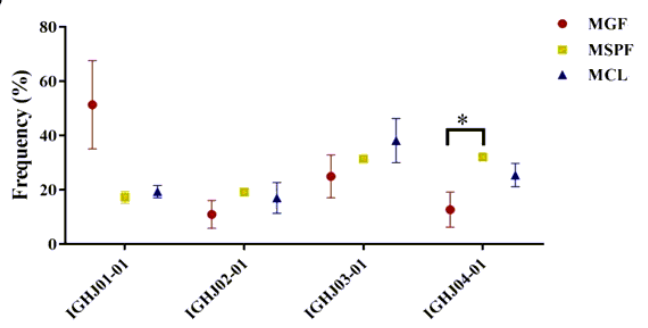

IGHJ GenName mapped genes(total)

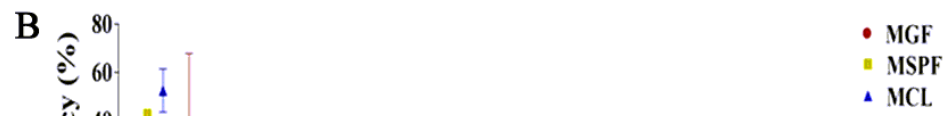

MSPF
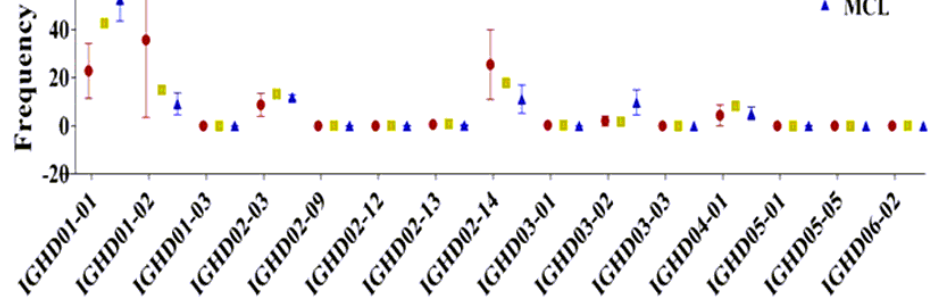

IGHD GenName mapped genes

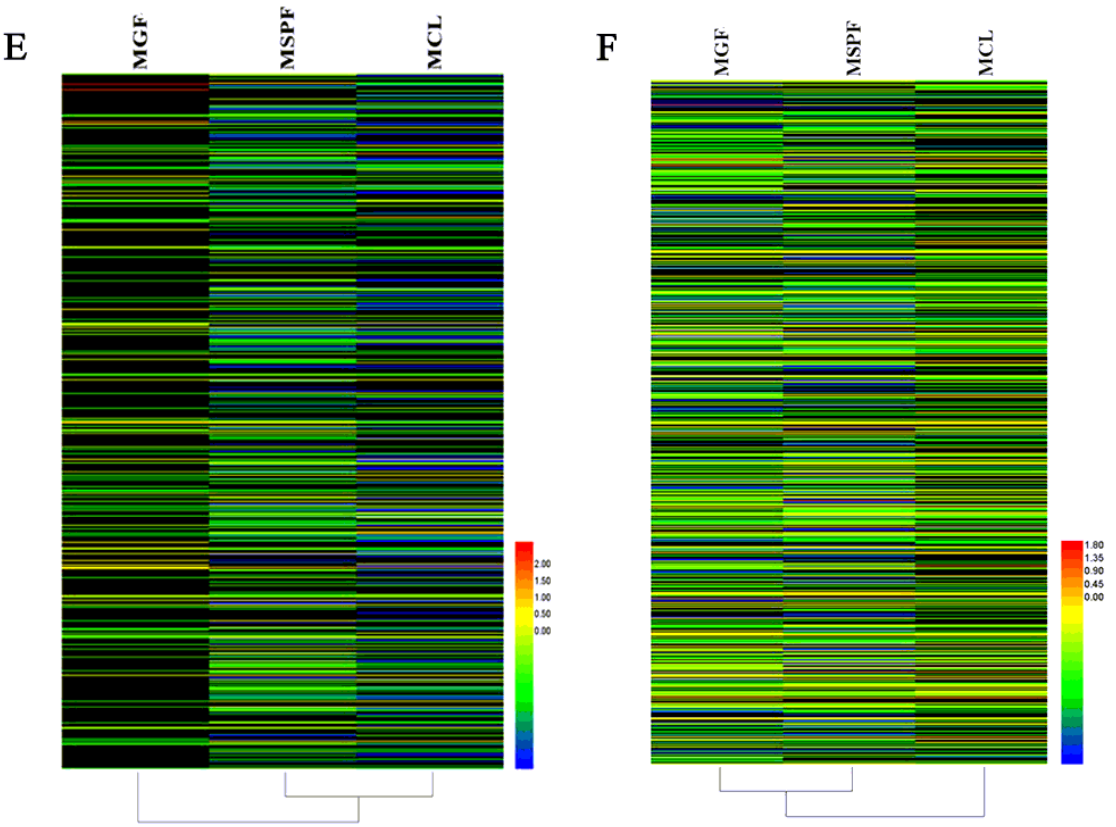

Figure 4 
Distinct usage frequency of IGHD, IGHJ and IGHV-IGHJ gene segments in the BCR heavy chain CDR3 region. A: The usage frequency of IGHD in intestinal total B cells; B: The usage frequency of IGHD in intestinal memory B cells; C: The usage frequency of IGHJ in intestinal total B cells; D: The usage frequency of IGHJ in intestinal memory B cells; E-F: The combination frequency of IGHV-IGHJ genes in intestinal total and memory B cells. MGF: Germ-free mice; MSPF: Specific Pathogen-free mice; MCL: Clean mice.
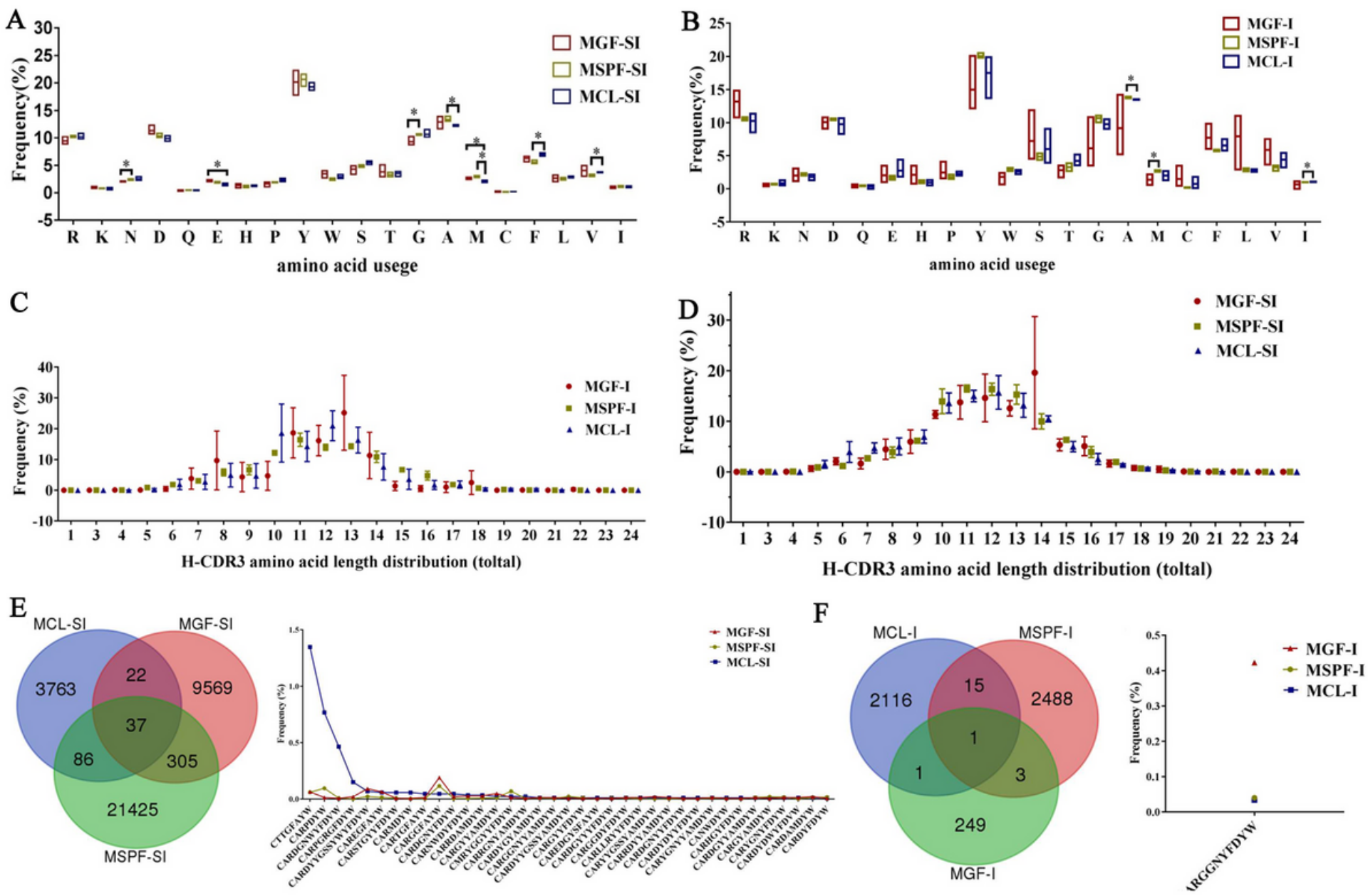

Figure 5

Amino acid sequence analysis of BCR heavy chain CDR3 region. A: Animo acid usage in intestinal total B cells; $B$ : Animo acid usage in intestinal memory B cells; C: Animo acid length distribution in intestinal memory B cells; D: Animo acid length distribution in intestinal total B cells; E: Overlap animo acid among GF, SPF and CL mice in intestinal total B cells; F: Overlap animo acid among GF, SPF and CL mice in intestinal memory B cells. SI: Total B cells; I: Memory B cells; MGF: Germ-free mice; MSPF: Specific Pathogen-free mice; MCL: Clean mice. 


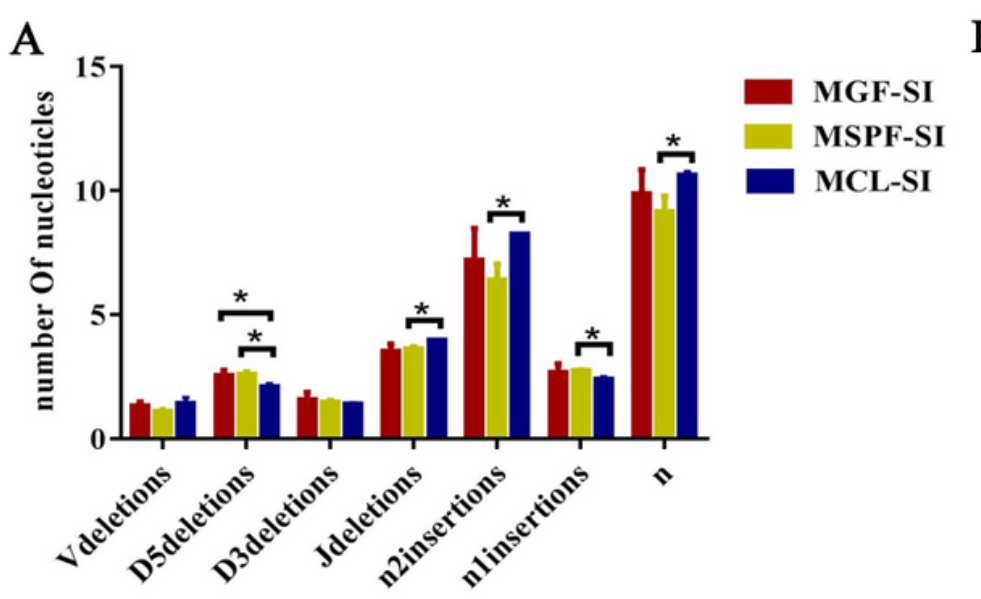

C

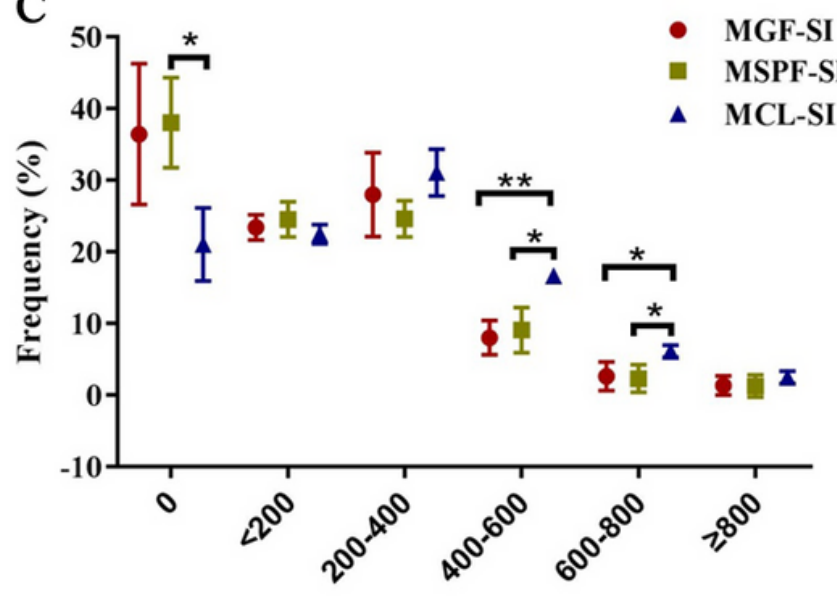

$I G H V$ mutation per 10000 bases

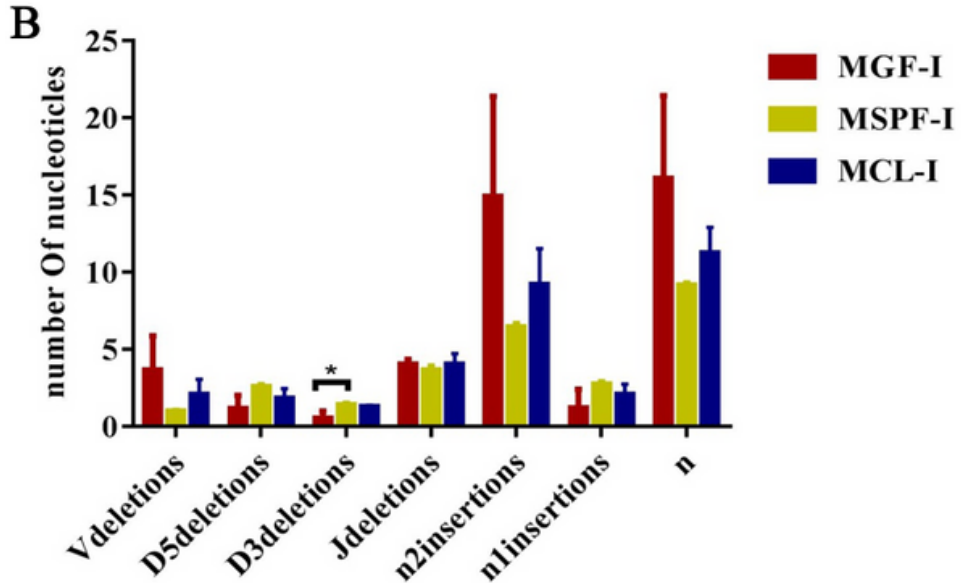

D

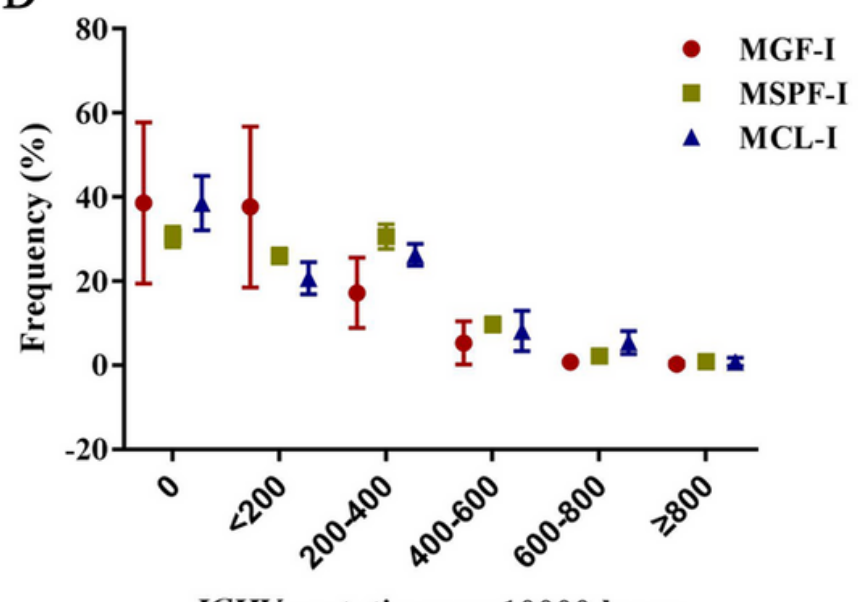

$I G H V$ mutation per 10000 bases

Figure 6

Mutation detection of BCR heavy chain CDR3 region. A-B: Deletion and insertion of BCR CDR3 sequence in intestinal total and memory B cells; C-D: High frequency mutations rate of BCR CDR3 sequence in intestinal total and memory B cells. SI: Total B cells; I: Memory B cells; MGF: Germ-free mice; MSPF: Specific Pathogen-free mice; MCL: Clean mice.

\section{Supplementary Files}

This is a list of supplementary files associated with this preprint. Click to download.

- Additionalfile.docx

- Arrivechecklist.pdf 